\title{
Respiration of Fruits*
}

\section{Mechanism of Respiration}

$\mathrm{D}^{\mathrm{u}}$ URING respiration, the entrance of oxygen and its distribution through the tissues to the individual cells of a fruit, also the reverse processes involving the giving off of carbon dioxide, take place by diffusion. There is a fine network of channels ramifying through the tissues to aid such gaseous interchange. The diameter of the pulp cells of the full-grown apple is about $150 \mu$, and of the channels about $5 \mu$. The surface of interchange is very large, being a square metre for a single fruit. The intercellular space is 10-20 per cent of the whole tissue. In such a system as this, there must be gradients of gaseous concentration; though it can be shown that such gradients are very small.

The peel of the apple plays an important part in regulating gaseous interchange between the fruit tissues and the atmosphere. The cells of the peel are smaller and more compact than those of the parenchyma beneath, and their outer walls are covered with a thick cuticle which is comparatively impermeable to gases. But the peel is broken by a system of pores, which constitute a path of gaseous interchange. That the peel constitutes a resistance to the path of gaseous interchange can be shown by setting up two apples with 100 per cent atmospheres of carbon dioxide at their centres, one apple with the skin removed and the other with the skin intact. The escape of carbon dioxide observed will be much greater in the former. Owing to the resistance to diffusion offered by the peel, the composition of the internal atmosphere differs considerably from that of air. Conditions can easily arise which lead, through a conjunction of low skin porosity and high respiratory activity, to concentrations of carbon dioxide inside the fruit which are directly injurious. So much has been definitely shown, but so far no thorough study of the internal atmosphere in relation to the variables affecting it during growth and storage has been carried out.

\section{Changes in Respiratory Activity}

Throughout its life-history, the apple shows distinct changes in respiratory activity. These may be correlated with the five morphological and physiological stages which occur during the development of this fruit. First, there is the stage of cell division which lasts from fertilisation until about three or four weeks afterwards, when the fruit is about the size of a walnut. This goes on until about a hundred million cells are formed

- Substance of the Friday evening discourse delivered by Dr. Franklin Kidd at the Royal Institution on November 9, 1934. in a typical fruit, after which the number of cells does not appreciably increase. The enlargement of these cells constitutes the second stage. Then the fruit matures and produces aroma and flavour. This is the third stage. Now the fruit is ripe, and under natural conditions it falls from the tree. The fourth stage follows as a longer or shorter period of senescence. The fifth stage is the functional breakdown culminating in death.

Respiratory activity is greatest at the beginning of fruit formation; at the end of cell division (first stage) it has fallen to about one fifth of its original value. During cell enlargement (second stage), respiration continues slowly to decrease. During the process of maturation (third stage), a critical change in respiratory activity occurs in that there is a sharp rise. This may be called the climacteric. Senescence (fourth stage) sees a slow decline from the peak of respiratory activity reached during the climacteric. Finally, at death (fifth stage) there is a sudden rise followed by a fall to zero.

\section{Chemistry of Respiration}

The most important substances connected chemically with the respiration of the apple fruit are sugars (fructose, glucose and sucrose), acids (mainly malic acid), starch and proteins.

During the first stage, the proteins are the chief constituent of the practically non-vacuolate cells. During cell enlargement, sugars accumulate from about 1 per cent to about 9 per cent fresh weight. Vacuoles appear and occupy about 80 per cent of the cell volume. In these, most of the sugars collect. It is significant that fructose and sucrose are the sugars the concentration of which rises, while glucose remains relatively low and steady in concentration. Starch accumulates in the cytoplasm up to about the middle of this stage, after which it diminishes to zero.

Tissue oxidations are associated with enzyme catalysis, determined by (1) enzyme content and (2) content of respirable material. So, to be more exact, the standard of reference should not be unit fresh weight as heretofore used, but unit living matter, thus omitting vacuole and cell wall. Considered thus, the fall during the first period is smaller and may be related to a decrease in enzyme activity. In the second stage an increase in respiration occurs, due to the accumulation of the necessary fuel sugars.

If fruit is gathered during the second stage of growth, respiration rapidly falls. Then there is a pause, followed by a continuation of the decrease. The respiratory activity then settles to a steady 
decline. This is followed in due course by the climacteric rise, the senescent decline, and the final burst of activity falling away to zero (Fig. 1).

The chemical changes in fruit gathered at this stage and stored are as follows: As soon as the fruit is gathered, the starch content declines and glucose content naturally rises. But, surprisingly, fructose, and sucrose also, rise. The end of the pause in initial fall of respiratory activity marks the final disappearance of starch.

As soon as starch is no longer present, the sucrose begins to disappear, the total fructose content remaining steady, while the total glucose content falls. Unlike starch, however, sucrose does not completely disappear. These changes are con-

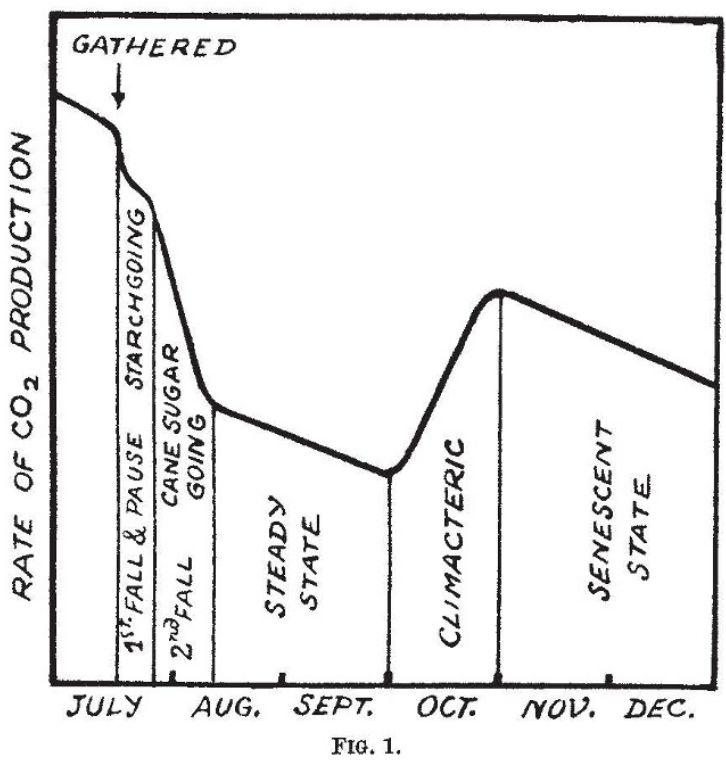

sidered to complete the phase of adjustment to starvation conditions (Fig. 2).

There follows a period the only marked features of which are a slow decline in the total glucose content and the sucrose content. At the climacteric, fructose begins to disappear for the first time. At the same time, sucrose rises ; thus paralleling the increase in respiration. The concentration of cane sugar and the rate of respiration reach a maximum together at the peak of the climacteric and thereafter decline together.

In explanation of these changes, the heterogeneity of the cell system and the probability that respired sugars are not equally distributed must be taken into account and also the fact that, after gathering, the cells are changing from a state of growth, and of supply and accumulation, to one of cessation of growth and of supplies. The theory advanced is that the sugar respired is active or $\gamma$-fructose. Other things being equal, the rate of respiration is determined by the concentration of this active sugar in the eytoplasm. The principal reactions determining concentration of active fructose are :

(I) Glucose $\rightleftarrows$ active fructose $\rightarrow$ inactive fructose or other normal hexose (vacuole).

(II) Glucose + activefructose $\rightleftarrows$ sucrose (vacuole); and the seat of these reactions is thought to be at the interface between the cytoplasm and the vacuole. It is further suggested that prior to the climacteric, sucrose and normal fructose do not as such penetrate from the vacuole into the cytoplasm. When the fruit is on the tree, glucose arrives at the cell wall and passes aoross the cytoplasm through a declining diffusion gradient. At certain points in the eytoplasm any excess of glucose above a certain concentration is condensed to starch. At the interface between cytoplasm and vacuole the conversion of some of the glucose into active or $\gamma$-fructose takes place, and the formation of cane sugar occurs.

The theory accounts for the accumulation of sucrose and fructose in the vacuole during growth and supplies an answer to the questions: Why, after gathering, are the cells unable to maintain a high concentration of cane sugar? Why, nevertheless, does the concentration of cane sugar rise after gathering so long as starch is present? Why, finally, does a steady low concentration of cane sugar occur instead of a complete disappearance as in the case of starch?

With the onset of the climacteric, it is supposed that the interface between vacuole and cytoplasm becomes permeable to normal fructose. This change in permeability is connected with the inner surface of the cytoplasm and possibly with the attainment of a critical hydrogen ion concentration in the vacuole. The acidity of the vacuole is due to the organic acid present. Whether the apple remains on the tree or is gathered, the acidity of the vacuole steadily falls from the beginning of the cell enlargement stage at approximately the same rate. This explains why the climacteric rise oceurs very nearly at the same time, irrespective of date of gathering, since the acidity falls in any case, at about the same time, to a certain critical level at which the climacteric occurs. Other evidence has been obtained that the acidity of the vacuole is the main factor in determining the onset of the climacteric.

\section{Storage of Frutts}

The climacteric marks the beginning of the production of odour and flavour. Experiment has shown that a substance is evolved during this stage-a substance toxic to seeds and destroyed by bromine. This substance is produced in such small quantities that its identification is not easy ; but there are reasons for believing that it is the gas ethylene. The biological effects of the vapour 
from ripe apples and of ethylene on other plants are the same. Both, for example, cause an immediate rise in the respiratory activity of other unripe fruit, and this rise bears every resemblance to the true climacteric rise of ripening. Thus unripe fruits exposed to the vapour of ripe fruits begin ripening at once. Such 'autocatalysis' can be prevented by treating air passing from the ripe fruit with ozone.

Among other substances produced at the climacteric is a dense, slowly diffusing vapour which causes the browning of the skin of the apple. Such deterioration can be avoided by very rapid ventilation with fresh air. A more practical way, however, is to wrap the apple, during the climacteric, in tissue paper impregnated with an odourless mineral oil.

The production of such gases during the climacteric is also responsible for increased susceptibility in apples to low temperature injury when kept in cold storage. How far the origin of these toxic substances can be traced to fructose is not yet known.

\section{Intermediate Stages of Respiration}

Fruit can produce carbon dioxide in the absence of oxygen by respiration. Blackman and Parija have shown that the initial rate of such fermentative breakdown in a ripe apple is a function of the oxygen supply prior to its removal to anærobic conditions (nitrogen atmosphere). They therefore concluded that the first stages of sugar breakdown are anærobic in any case, and that oxygen has a stimulating effect on the supply of this sugar. But this theory, though it explains the decline in respiration after removal of the fruit to anærobic conditions, does not explain why such decline is not to zero but to a constant level of activity. Thus, although oxygen stimulates supply of respiratory sugar, it is not essential to it.

Trout, realising that acetaldehyde is given off by ripe apples, and that this substance is also a recognised intermediate product during the fermentation of sugars, examined the effect of supplying acetaldehyde artificially to fruits. When supplied slowly, the added aldehyde quickly became oxidised. On the other hand, when supplied quickly there was a definite rise in aldehyde content and at the same time ethyl alcohol appeared in the tissues. The two processes of oxidative and fermentative breakdown appeared to be proceeding together with a consequent increase in rate of sugar loss. From this, Trout suggested that the fermentative process takes place as far as the acetaldehyde stage irrespective of oxygen present. After that, if much oxygen be present, the aldehyde is oxidised to carbon dioxide and water ; or, on the other hand, if no oxygen is present, it reacts with methyl glyoxal, formed in the first splitting of the sugar. The former process must be the more rapid one, and therefore in the presence of oxygen the concentration of aldehyde in the tissues is low. When it is artificially raised, the reaction with methyl glyoxal occurs and the results obtained are to be expected. If this hypothesis be correct, glycerol must be formed; but, since it is known not to accumulate, we must assume, following the Blackman theory of oxygenanabolism, that glycerol is the substance which is re-synthesised to sugar.

Experimental evidence suggests that the removal of oxygen from apples in the post-climacteric state is the elimination of the factor which produces the

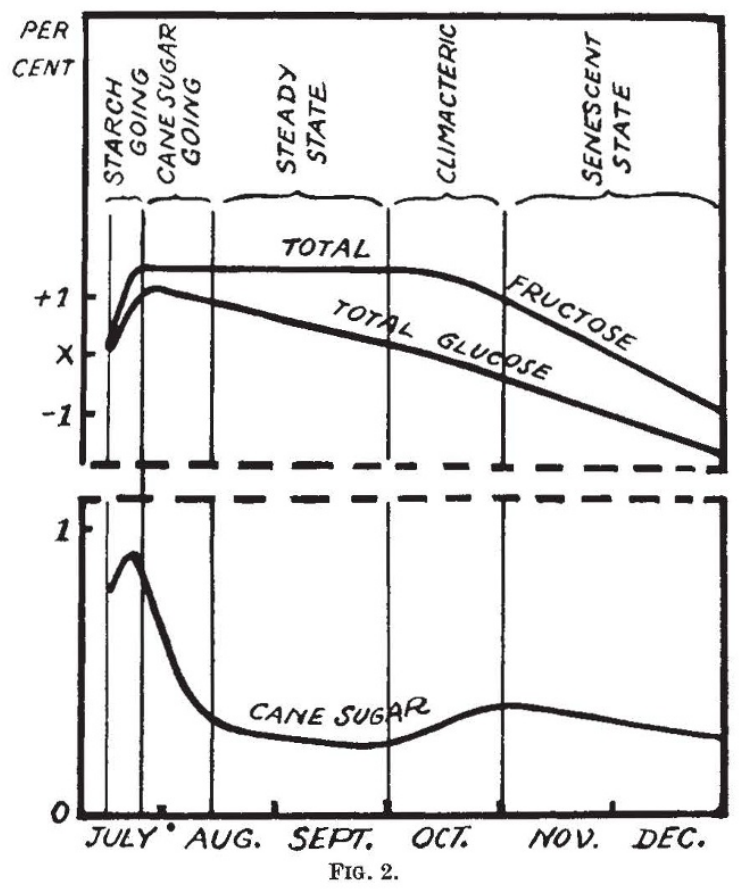

climacteric rise, that is, the entrance of fructose from the vacuole. It is therefore suggested that Blackman's theory of the activation of sugar by oxygen during respiration applies only to the vacuolar fructose, and that activation is really the entry into the cytoplasm for which the presence of oxygen is essential. If this is the case, then the climacteric rise should not occur in the absence of oxygen. There is evidence that such is the case.

During senescence, a slow decline in respiration occurs. Aldehyde and alcohol increase during this period, and it is suggested that this is due to diminishing activity of the system for the oxidation of aldehyde by molecular oxygen. Indeed, ageing apples become increasingly sensitive to lack of oxygen. Quite a small decrease in amount of oxygen in the atmosphere causes fermentation in apples which are in an advanced state of senescence. 


\section{Respiration and Life Duration}

Fruits of a short life-cycle, such as the strawberry, exhibit a higher respiratory activity than fruits of a longer life-cycle such as the apple. Variation occurs, however, even within a species. For example, in apples, the variety Bramley's Seedling respires at about two-thirds the rate of the Worcester Pearmain. The life duration of the latter is about one and a half times that of the former. Respiratory activity also varies with the nutrition of the fruit during growth. Therefore, if respiratory activity during cultivation of the fruit be increased by increasing the nitrogen content of the soil, life during storage is shortened, and vice versa. A more striking example, however, is the result obtained by gathering fruits at various stages during the second period of growth. At the beginning of this period, the cytoplasm and the cane sugar per cell are at their minimum, and so therefore is respiratory activity. Fruit gathered at this stage will thus have a longer life during storage than fruit gathered at any subsequent stage.

A young apple, in spite of the fact that it contains little carbohydrate when gathered, loses more carbon before death than does a full-grown fruit. A full-grown fruit does not usually lose more than $0 \cdot 2-0.4$ per cent of its carbohydrate and acid prior to death. It appears likely that it is the 'rate of living' which kills and that the machine breaks down from wear and tear and not from lack of fuel.

From these observations, three new agencies have come into use for the purpose of controlling the span and speed of life in fruits, namely : (l) use of ethylene as an accelerator of ripening; (2) use of carbon dioxide as a depressor of respiratory activity, thus retarding ripening and lengthening life; (3) the use of atmospheres poor in oxygen, thus reducing respiratory activity, delaying the climacteric and retarding ripening.

The possible use of ozone in controlling ripening is still in the experimental stage.

\section{The Teaching of Optics*}

$\mathrm{O}$ PTICS and mechanics are twin Cinderellas in the teaching of elementary physics. The beginner finds with growing disappointment that each covers ground remote from those thrilling matters of real life that they promised to deal with-remote from engines and machinery and from real optical instruments such as telescopes and microscopes. To make matters worse, a confusing fight rages round the teaching of each : a battle of units in mechanies, in which the mass and the weight of the projectiles employed seem inextricably mixed, and a battle of signs in optics, with $1 / v-1 / u=1 / f$ and $1 / v+1 / u=1 / f$ as its war cries. Mechanics has been rescued by the toy manufacturers-if we may call admirable constructional apparatus such as 'Meccano' toysand the mathematical studies of mechanics which seem so artificial if attacked too early are left until the later school stages when they can bring a real delight unspoiled by disappointed hopes of romance.

Optics may follow a similar path with constructional sets of lenses and mirrors as Christmas toys to provide the practical delights that formal teaching often misses ; and for several years such sets have been on sale, with optical components and adjustable framework for making a variety of working optical instruments. We grudge no toyshop such excellent wares, yet we cannot help

- Report of the Committee appointed by the Physical Society to consider and make Recommendations on the Teaching of Geometrical Optics. Pp. $v+86$. (London: Physical Society, 1934.) 68. net. feeling that elementary optical teaching is at fault and should itself capture and use some of the thrills that belong to actual instruments, before attempting to build a formal structure-an attempt long ago condemned in other branches of teaching. Many teachers, feeling this, now begin optics with a course that is not merely simplified but wholly changed in order and emphasis; yet even they must feel the drag of examination demands, textbook styles and the weight of tradition. So it is pleasing indeed to find that the Physical Society's Report urges that "The early approach to lens and mirror optics is best made along experimental lines, and every effort should be made to implant a sound conception of the main physical phenomena of image formation before mathematical formulæ are introduced", and suggests that an experimental start, free from mathematics, might be made with, "for example, elementary illumination and photometry, and the action of simple instruments such as the telescope, microscope and projection lantern". The Report says regretfully, "In only too many cases the beginning and end of optical instruction in schools seems to be bound up with $1 / v-1 / u=1 / f^{\prime \prime}$, a type of formula which, as one of the authors says later in the Report, is not much used in real optical work !

While the Report expresses these hopes for the future of elementary teaching, its main concern is with the conventions of signs in optical formulæ. 